\title{
Pretreatment of Total Phosphorus Content in Saline Soil in Arid Area by Fractional Differential Algorithm
}

\author{
Cheng-Biao Fu, ${ }^{1,2}$ Shu Gan, ${ }^{1 *}$ Xi-Ping Yuan, ${ }^{1}$ \\ Hei-Gang Xiong, ${ }^{3}$ and An-Hong Tian ${ }^{1,2}$ \\ ${ }^{1}$ Faculty of Land Resource Engineering, Kunming University of Science and Technology, \\ Kunming, Yunnan 650093, China \\ ${ }^{2}$ College of Information Engineering, Qujing Normal University, \\ Qujing, Yunnan 655011, China \\ ${ }^{3}$ College of Applied Arts and Science, Beijing Union University, \\ Beijing 100083, China
}

(Received March 28, 2018; accepted August 8, 2018)

Keywords: total phosphorus content, spectral data pretreatment, correlation coefficient, fractional differential

There are few reports on the pretreatment method based on a fractional differential algorithm for the total phosphorus content in saline soil. Therefore, the desert soil in Fukang City of XingJiang, China, was chosen as the research focus. Field hyperspectral data and surface soil down to $20 \mathrm{~cm}$ depth were collected between May 9-23, 2017. The Grünwald-Letnikov fractional differential algorithm was introduced to explore the pretreatment effect of the correlation between total phosphorus content and five spectral transformations. Simulation results showed that this fractional differential algorithm could elaborately describe the slight differences in spectral reflectance, and the fractional differential was close to zero on the whole. Because the sensitivity of the spectrum is controlled by the weighted order, when the order gradually increased from zero order to first order, the curve of the fractional differential slowly approached the first-order differential curve, and it slowly approximated the secondorder differential curve while increasing from first order to second order. The overall trend in band number that satisfied the 0.05 significance test under five spectral transformations was an initial increase followed by decrease as the order increased. The maximum absolute values of correlation coefficients appeared at 1.2 and 1.4 order; the corresponding band was $2067 \mathrm{~nm}$. This study made up for the research gap in field measurement spectra based on the fractional differential algorithm, and revealed a novel preprocessing method for field hyperspectral data.

\section{Introduction}

Because the spectral reflectance curve differs from the traditional first order and second order curves after integer-order differential processing, there are obvious shortcomings of the pretreatment method based on the traditional integer-order differential algorithm, which

*Corresponding author: e-mail: sgbf@kmust.edu.cn

https://doi.org/10.18494/SAM.2018.2047 
disregards the gradual fractional differential information between the 0th-order curve and the first- or second-order curve by considering only the preprocessing method of integerorder differentials. ${ }^{(1)}$ Consequently, it may result in the loss of important information and the degradation of modeling accuracy.

In the past few years, the fractional derivative has been introduced into the field of spectral analysis by domestic and foreign scholars. Kharintsev and Salakhov used the fractional differential algorithm to separate overlapping spectral features, ${ }^{(2)}$ as it has some effectiveness in spectral analysis applications. Tong et al. improved the algorithm of the integer Savizzy-Golay differential by using the fractional derivative formula. The Savitzky-Golay differential was found to be better than integer order. ${ }^{(3)}$ Zhang et al. utilized the Grünwald-Letnikov fractional differential to preprocess spectral reflectance data of saline soil in the two regions: Yutian and Ebinur Lake in the Xinjiang Uygur Autonomous Region. Their results showed that the fractional differential could refine the changing trend of the correlation coefficient, standard deviation, and information entropy, and provided a reference for feature band selection and other applications. $^{(1)}$ Wang et al. took Ebinur Lake as the research target, differentiated the spectral reflectance data of soil in steps of 0.2 intervals using the Grünwald-Letnikov fractional differential formula. Partial least squares regression (PLSR) was adopted to estimate the soil organic matter content. The 1.8-order differential model of the original spectrum was optimal, the coefficient of determination of validation reached 0.91 , the root mean square error was 2.70 , and the ratio of performance to deviation (RPD) achieved 3.42, which indicated that the 1.8-order differential model has a better prediction capability. ${ }^{(4)}$

However, the fractional differential algorithm described above has mainly been used to preprocess diesel fuel, soil salt content, and soil organic carbon content. At present, the application of the fractional differential algorithm to estimate total phosphorus content has not been reported. Phosphorus in soil is a limiting and common nutrient element required for plant growth. ${ }^{(5,6)}$ Traditional laboratory chemical analysis methods have the disadvantages of destroying collected samples, and of being time-consuming, labor intensive, and high in cost. ${ }^{(7,8)}$ Visible/near-infrared reflectance spectroscopy can be applied to estimate soil total phosphorus content because it is fast, efficient, and economically advantageous. ${ }^{(9,10)}$ In this study, we attempt to use the Grünwald-Letnikov fractional differential algorithm to preprocess the desert soil total phosphorus content without human interference in the field, to investigate the preprocessing effect on hyperspectral data using the fractional differential algorithm, and to determine the best fractional differential order. Results provide a reference for the rapid acquisition of soil nutrient parameters in precision agriculture using hyperspectral remotesensing methods, and indicate that the requirements of modern precision agriculture regarding soil nutrient real-time detection have been met. This method makes up for the deficiency of visible-light and near-infrared spectroscopy in soil total phosphorus analysis, improves the estimation accuracy of soil total phosphorus content, and provides effective support for quantitative estimation of the total phosphorus content in desert soils. 


\section{Materials and Methods}

\subsection{Soil spectrum acquisition and total phosphorus content test}

The study area was located at the northern edge of the eastern Tianshan Mountains in Xinjiang and the southern edge of Junggar Basin, at east longitude $87^{\circ} 44^{\prime}-88^{\circ} 46^{\prime}$ and north latitude $43^{\circ} 29^{\prime}-45^{\circ} 45^{\prime}$. Field investigations confirmed that the research area was far from any human settlements and had not been affected by human activities. The surface soils basically retained the original ecological features. In the study area, five sampling lines (between 600-800 m) were arranged from south to north, and five sampling points (between $300-500 \mathrm{~m}$ ) were distributed on each sampling line, so there was a total of 25 sampling points in all. Field spectra of soil samples was conducted from 9 to 23 May 2017 using the Field spec ${ }^{\circledR}$ $3 \mathrm{Hi}$-Res spectrometer, which had a wavelength range of 350-2500 nm. $0-20 \mathrm{~cm}$ soil layers were acquired on the surface. Soil samples were placed in a sealed bag, brought back to the laboratory for natural air drying, impurity removal, grinding, sieving, and other pretreatments, and then sent to the Xinjiang Institute of Ecology and Geography, Chinese Academy of Sciences, for the determination of soil total phosphorus content by professionals.

\subsection{Grünwald-Letnikov fractional differential algorithm}

There are many fields involving fractional calculus theory and applications, mainly including control systems, nonlinear dynamics, biomedicine, and digital signal processing. ${ }^{(11)}$ The commonly used Grünwald-Letnikov fractional differential is defined as

$$
{ }_{a} D_{t}^{v} f(x)=\lim _{h \rightarrow 0} f_{h}^{v}(x)=\lim _{\substack{h \rightarrow 0 \\ n h \rightarrow t-a}} h^{-v} \sum_{r=0}^{n} C_{r}^{-v} f(x-r h),
$$

where

$$
C_{r}^{-v}=\frac{(-v)(-v+1) \cdots(-v+r-1)}{r !} .
$$

According to the definition Eq. (1), if the duration of the function $f(x)$ is $x \in[a, t]$, the signal duration is equally divided with interval $h=1$; then $n$ can be described as

$$
n=\left[\frac{t-a}{h}\right]^{h-1}=[t-a]
$$

The difference expression of the fractional differential of function $f(x)$ can be shown as 


$$
\begin{gathered}
\frac{d^{v} f(x)}{d t^{v}} \approx f(x)+(-v) f(x-1)+\frac{(-v)(-v+1)}{2} f(x-2) \\
+\frac{(-v)(-v+1)(-v+2)}{6} f(x-3) \\
\ldots+\frac{\Gamma(-v+1)}{n ! \Gamma(-v+n+1)} f(x-n) .
\end{gathered}
$$

\subsection{Spectral data processing}

Firstly, the spectrum was smoothed by the Savitzky-Golay method. Secondly, spectral reflectances in the range of 350-399 and 2401-2500 nm were eliminated because the signalto-noise ratio was very low, and are shown in Fig. 1. Then, the bands located in the moisture absorption bands (1355-1410 and 1820-1942 nm) were also excluded. These are shown in Fig. 2(a). The reflectance spectroscopy data of the 400-1354, 1411-1819, and 1943-2400 nm bands, shown in Fig. 2(b), were used in the study. Finally, in order to eliminate the influence of background and atmospheric scattering and to improve the contrast among different absorption characteristics, it was often necessary to perform various transformations on original hyperspectral data. The original spectral reflectance $R$ was transformed into four different forms: reciprocal $(1 / R)$, logarithmic $(\lg R)$, logarithmic reciprocal $(1 / \lg R)$, and root mean square $(\sqrt{R})$.

\section{Experimental Results and Discussion}

\subsection{Computational characteristics of fractional differential in $R$}

Taking the average of the original spectrum $(R)$ as an example, a total of 11 order differentials were obtained at intervals of 0.2 order. The range of the $1140-1340 \mathrm{~nm}$ band was

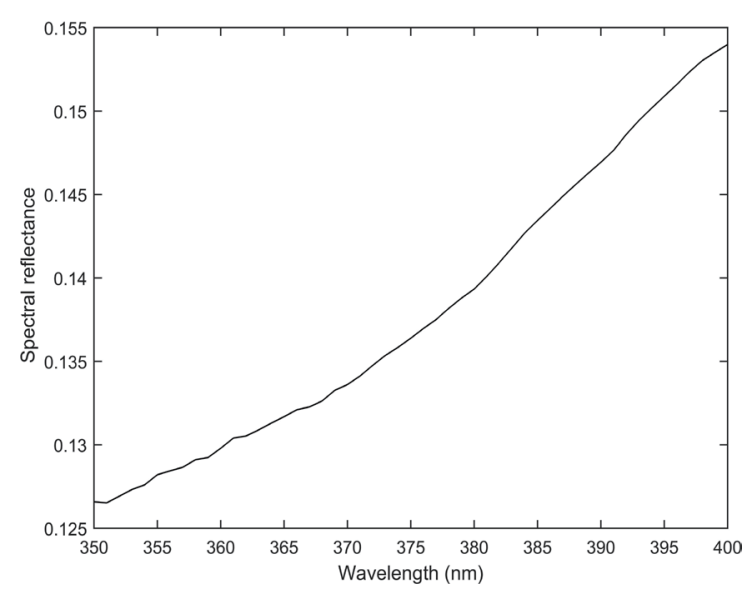

(a)

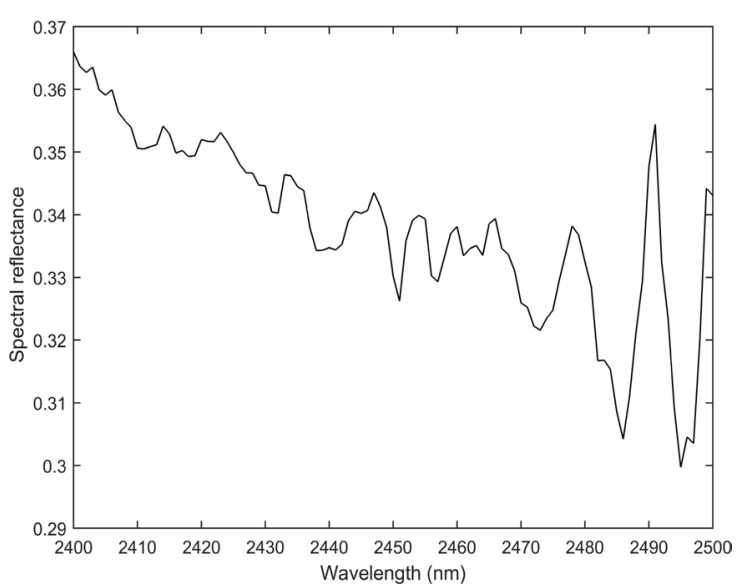

(b)

Fig. 1. Edge band spectral curve: (a) 350-399 and (b) 2401-2500 nm. 


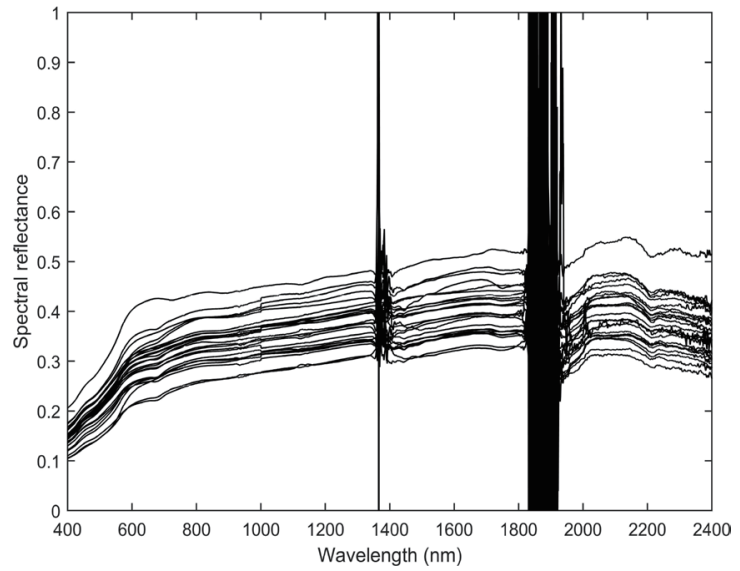

(a)

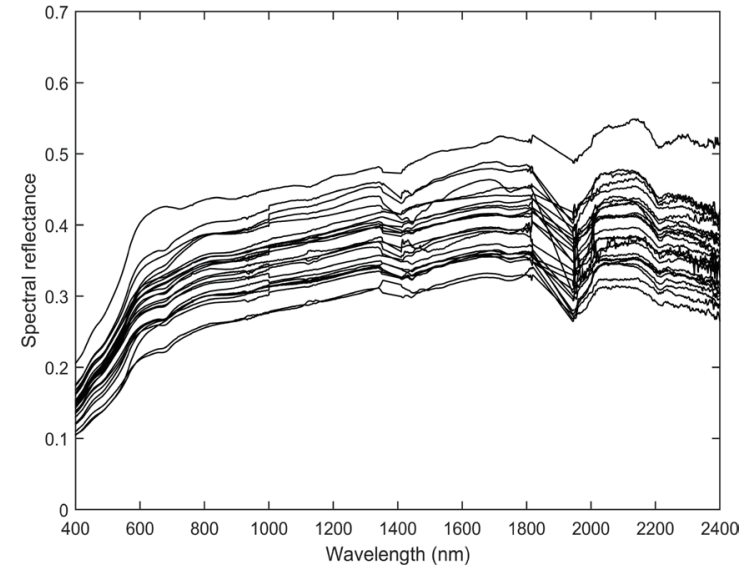

(b)

Fig. 2. Spectral reflectance curves: (a) full band and (b) moisture-deleted absorption bands.

selected to show the results of the fractional differential calculation in Fig. 3. The variation tendency of $R$ gradually approached the first-order differential curve while increasing from zero order to first order. When the order was increased from first order to second order, the variation tendency of $R$ slowly approximated the second-order differential curve. It was reported that the fractional differential is close to the zero value after processing by the second-order differential in Fig. 3.

\subsection{Correlation coefficient change}

In the process of total phosphorus content model building, the correlation between total phosphorus content and spectral reflectance was chosen to determine the sensitive band. The stronger the correlation is, the more likely this band is to be selected as the sensitive band. A correlation coefficient greater than 0.5 could be viewed as moderate correlation. Therefore, taking $R$ as an example, the 11th-order differential of the correlation coefficient between total phosphorus content and spectral reflectance was analyzed and checked at the 0.05 significance level (threshold was equal to plus or minus 0.396). Simulation results are shown in Fig. 4. There is no band passing the 0.05 significance level in the range of 0 order to 0.4 order, and the band satisfied the 0.05 significance level from the 0.6 order as order increased.

\subsection{Bands satisfying $\mathbf{0 . 0 5}$ significance test under five spectral transformations}

The bands that satisfied the 0.05 significance test under five spectral transformations was calculated and are listed in Table 1 . It could be clearly observed that the spectral reflectance of various mathematical transformations satisfied the 0.05 significance test from the 0.6 order. In addition, the overall trend of band number was an initial increase and a later decrease as the order increased, but the corresponding order with the largest band number was not uniform; there were certain differences, such as $R$ and $1 / \lg R$ being of 1.4 order, $\sqrt{R}$ and $\lg R$ being of 1.2 order, and $1 / R$ being of 0.8 order. 


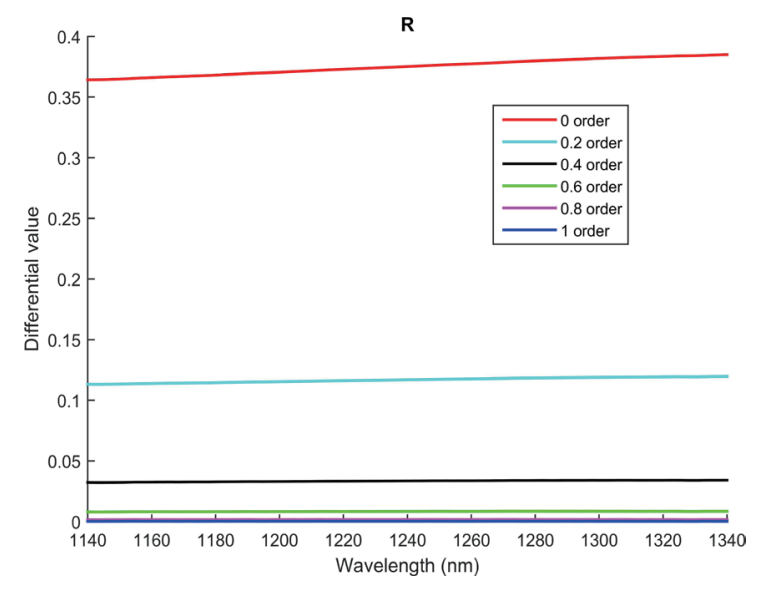

(a)

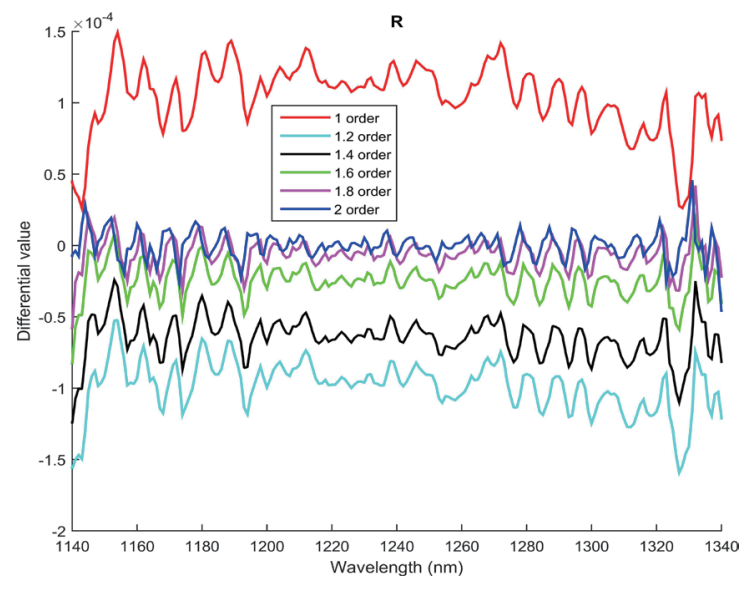

(b)

Fig. 3. (Color online) Fractional differential calculation result of R: (a) 0-1 and (b) 1-2 order.

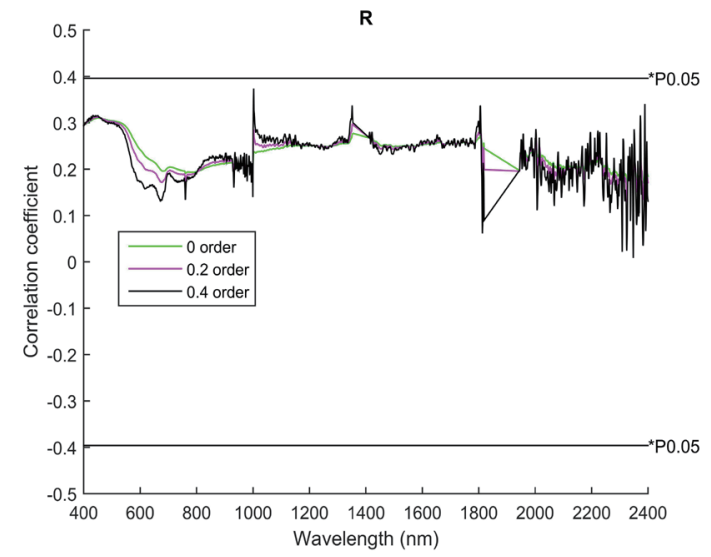

(a)

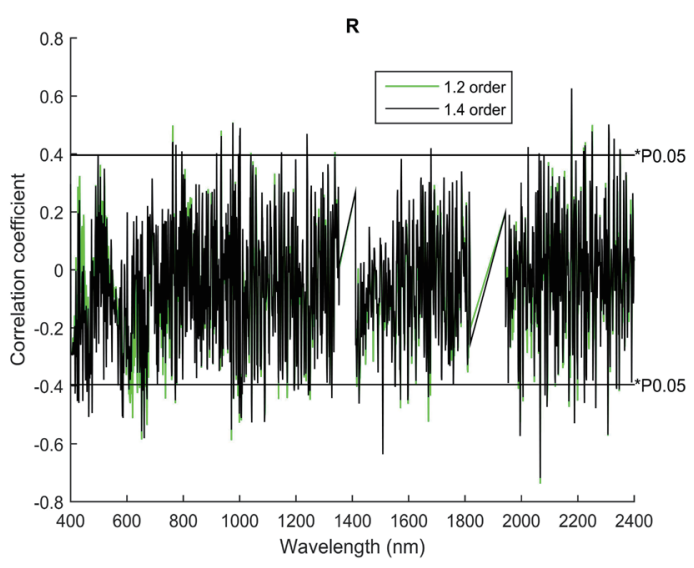

(c)

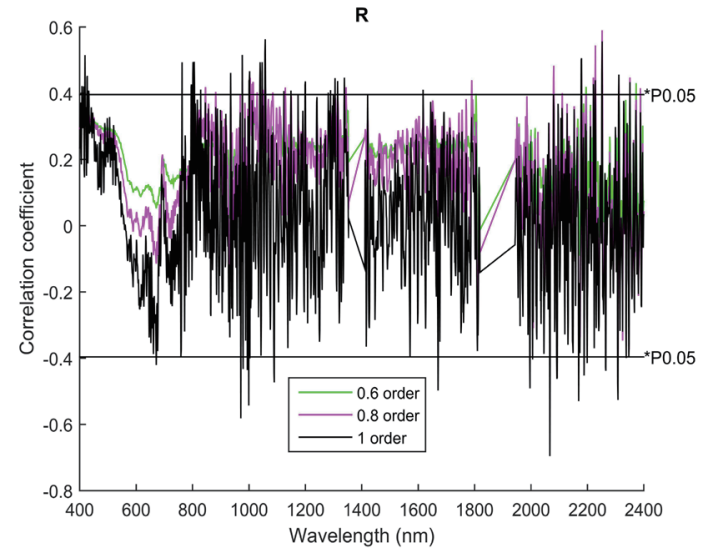

(b)

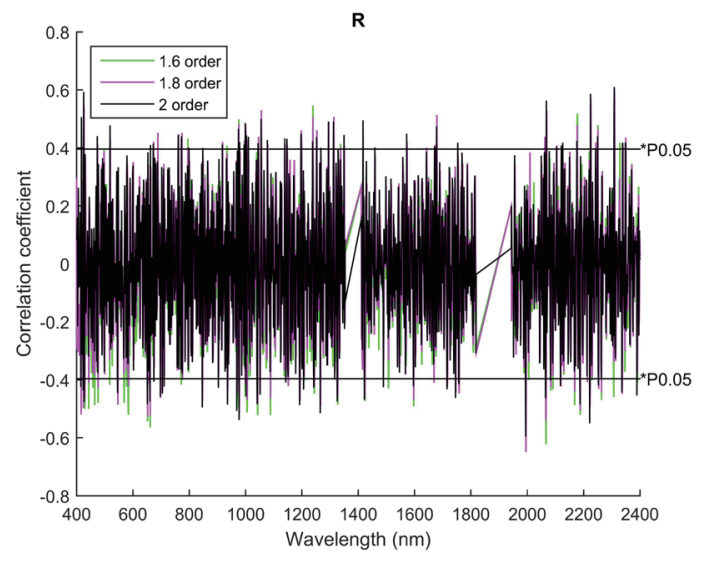

(d)

Fig. 4. (Color online) Change in correlation coefficient: (a) 0-0.4, (b) 0.6-1, (c) 1.2-1.4, and (d) 1.6-2 order. 
Table 1

Bands that satisfied the 0.05 significance test.

\begin{tabular}{lrrrrc}
\hline Order & $R$ & $\sqrt{R}$ & $1 / R$ & $\lg R$ & $1 / \lg R$ \\
\hline 0 & 0 & 0 & 0 & 0 & 0 \\
0.2 & 0 & 0 & 0 & 0 & 0 \\
0.4 & 0 & 0 & 0 & 0 & 0 \\
0.6 & 11 & 10 & 26 & 10 & 12 \\
0.8 & 46 & 50 & 193 & 55 & 44 \\
1 & 72 & 74 & 190 & 89 & 79 \\
1.2 & 92 & 107 & 147 & 119 & 74 \\
1.4 & 100 & 102 & 132 & 107 & 96 \\
1.6 & 95 & 97 & 109 & 101 & 87 \\
1.8 & 96 & 98 & 103 & 99 & 88 \\
2 & 97 & 93 & 100 & 98 & 89 \\
\hline
\end{tabular}

Table 2

Bands with the max absolute value of correlation coefficients under five transformations.

\begin{tabular}{llclccrcrcr}
\hline \multirow{2}{*}{ Order } & \multicolumn{3}{c}{$R$} & \multicolumn{2}{c}{$\sqrt{R}$} & \multicolumn{2}{c}{$1 / R$} & \multicolumn{2}{c}{$\lg R$} & \multicolumn{2}{c}{$1 / \lg R$} \\
\cline { 2 - 10 } & \multicolumn{1}{c}{ Max } & Band & \multicolumn{1}{c}{ Max } & Band & \multicolumn{1}{c}{ Max } & Max & Band & Max & Band & Max \\
\hline 0 & 0.310499 & 451 & 0.318157 & 451 & 0.338822 & 450 & 0.32543 & 451 & 0.308743 & 451 \\
0.2 & 0.312689 & 451 & 0.320503 & 451 & 0.342793 & 442 & 0.328006 & 442 & 0.310513 & 451 \\
0.4 & 0.373807 & 1002 & 0.373598 & 1002 & 0.373649 & 1002 & 0.373479 & 1002 & 0.376202 & 1002 \\
0.6 & 0.445148 & 1002 & 0.447989 & 1002 & 0.4565 & 1002 & 0.45086 & 1002 & 0.44373 & 1002 \\
0.8 & 0.590023 & 2252 & 0.587964 & 2067 & 0.585587 & 2067 & 0.591116 & 2067 & 0.584035 & 2252 \\
1 & 0.695528 & 2067 & 0.69587 & 2067 & 0.672586 & 2067 & 0.692454 & 2067 & 0.676921 & 2067 \\
1.2 & 0.737302 & 2067 & 0.733033 & 2067 & 0.700229 & 2067 & 0.725459 & 2067 & 0.731621 & 2067 \\
1.4 & 0.718571 & 2067 & 0.705614 & 2067 & 0.653319 & 1508 & 0.689809 & 2067 & 0.735853 & 2067 \\
1.6 & 0.631626 & 1995 & 0.631207 & 1995 & 0.6181 & 1995 & 0.62871 & 1995 & 0.64429 & 2067 \\
1.8 & 0.6489 & 1995 & 0.645911 & 1995 & 0.62003 & 1995 & 0.639893 & 1995 & 0.642674 & 1995 \\
2 & 0.610027 & 2309 & 0.60736 & 2309 & 0.628818 & 425 & 0.614293 & 425 & 0.602613 & 2309 \\
\hline
\end{tabular}

\subsection{Maximum absolute value of correlation coefficient}

The maximum absolute values of the correlation coefficient under five spectral transformations after 11th-order differential processing are shown in Table 2. $R, \sqrt{R}, 1 / R$, and $\lg R$ were of 1.2 order, corresponding to the $2067 \mathrm{~nm}$ band, and $1 / \lg R$ was of 1.4 order, corresponding to the $2067 \mathrm{~nm}$ band. At the same time, at the 1.2 order, maximum absolute values of correlation coefficients under five spectral transformations were greater than 0.7 .

\section{Conclusions}

The Grünwald-Letnikov fractional differential algorithm was applied to explore the pretreatment effect of field spectroscopy of saline soil that reflects the original ecological environment through four aspects. Simulations showed that pretreatment of fractional differentials could refine the subtle changes in the correlation between total phosphorus content and soil spectral reflectance. The correlation coefficient satisfied the 0.05 significance test and showed a trend of first increasing and then decreasing after fractional differential 
preprocessing. The maximum correlation coefficient under five spectral transformations all appeared in fractional orders. In this study, we explored the possibility of using the fractional differential algorithm to monitor soil total phosphorus content using the field hyperspectral data and provided a new perspective to predict total phosphorus content in arid areas.

\title{
Acknowledgments
}

This work was supported by the financial support of the National Natural Science Foundation of China (41861054, 41561083, 41671198) and Yunnan Province Science and Technology Department and Education Department Project (2017FH001-067, 2017FH001-117, 2016ZDX127), China.

\section{References}

1 D. Zhang, T. Tiyip, J. L. Ding, F. Zhang, I. Nurmemet, A. Kelimu, and J. Z. Wang: J. Spectrosc. 1 (2016) 1. https://doi.org/10.1155/2016/1081674

2 S. S. Kharintsev and M. K. Salakhov: Spectrochim. Acta, Part A 60 (2004) 2125. https://doi.org/10.1016/ j.saa.2003.11.013

3 P. J. Tong, Y. P. Du, K. Y. Zheng, T. Wu, and J. J. Wang: Chemometr. Intell. Lab. Sys. 143 (2015) 40. https:// doi.org/10.1016/j.chemolab.2015.02.017

4 J. Z. Wang, T. Tiyip, J. L. Ding, D. Zhang, W. Liu, and F. Wang: J. Spectrosc. 1 (2017) 1. https://doi. org/10.1155/2017/1375158

5 Y. T. Cheng, P. Li, G. C. Xu, Z. B. Li, S. D. Cheng, and H. D. Gao: Catena 136 (2016)175. https://doi. org/10.1016/j.catena.2015.02.015

6 C. A. Rosolem, A. Merlin, and J. C. L. Bull: Scientia Agricola 71 (2014) 309. https://doi.org/10.1590/01039016-2013-0345

7 A. Allbed, L. Kumar, and P. Sinha: Remote Sens. 6 (2014) 1137. https://doi.org/10.3390/rs6021137

8 J. Farifteh, A. Farshad, and R. J. George: Geoderma 130 (2006) 191. https://doi.org/10.1016/ j.geoderma.2005.02.003

9 N. Sahu, S. K. Singh, G. P. O. Reddy, N. Kumar, M. S. S. Nagaraju, and R. Srivastava: J. Indian Soc. Remote Sens. 44 (2016) 811. https://doi.org/10.1007/s12524-015-0540-7

10 C. H. Chen and C. H. Liou: Sens. Mater. 30 (2018) 365. https://doi.org/10.18494/SAM.2018.1753

11 G. S. M. Ngueuteu and P. Woafo: Mech. Res. Commun. 46 (2012) 20. https://doi.org/10.1016/ j.mechrescom.2012.08.003

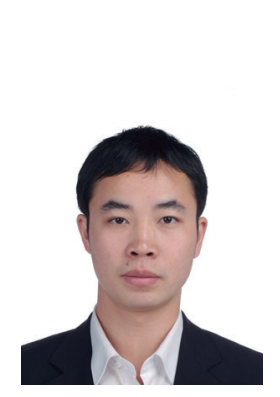

\begin{abstract}
About the Authors
Cheng-Biao Fu is a doctoral graduate student of the Faculty of Land Resource Engineering, Kunming University of Science and Technology. He received his B.S. and M.S. degrees from Chongqing University of Posts and Telecommunications, China, in 2005 and 2009, respectively. Since 2015, he has been a lecturer at Qujing Normal University. His research interests are in information identification and processing, hyperspectral remote sensing, and Internet of Things. (fucb@mail.qjnu.edu.cn)
\end{abstract}



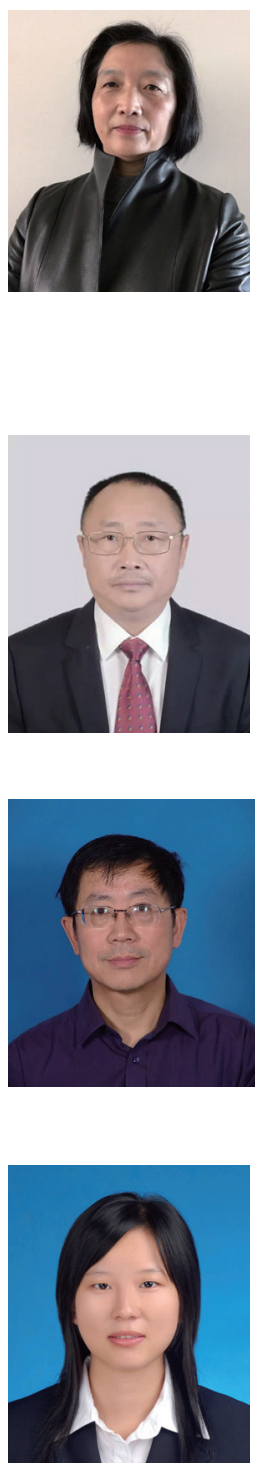

Shu Gan received her B.S. degree from Wuhan University of Surveying and Mapping Science and Tec, China, in 1987, her M.S. degree from Southwest Forestry University, China, in 1995, and her Ph.D. degree from Zhejiang University, China, in 2000. Since 2002, she has been a professor at Kunming University of Science and Technology. Her research interests are in spatial data modeling, surveying technology, and remote sensing.

(sgbf@kmust.edu.cn)

Xi-Ping Yuan received his B.S. degree from Wuhan University of Surveying and Mapping Science and Tec, China, in 1987, and he received his $\mathrm{Ph} . \mathrm{D}$. degree from Kunming University of Science and Technology, China, in 2007. Since 2003, he has been a professor at Kunming University of Science and Technology. His research interests are in surveying technology and remote sensing. (YXP@kmust.edu.cn)

Hei-Gang Xiong received his B.S. degree from Xinjiang University, China, in 1982, and his M.S. and Ph.D. degrees from Peking University, China, in 1988 and 1991, respectively. Since 1994, he has been a professor at Xinjiang University. Since 2004, he has worked in Beijing Union University. His research interests are in regional environmental sustainability, and remote sensing. (heigang@buu.edu.cn)

An-Hong Tian is a doctoral graduate student of the Faculty of Land Resource Engineering, Kunming University of Science and Technology. Since 2017, she has been an associate professor at Qujing Normal University. Her research interests are in remote sensing, and artificial intelligence. (tianah@mail.qjnu.edu.cn) 